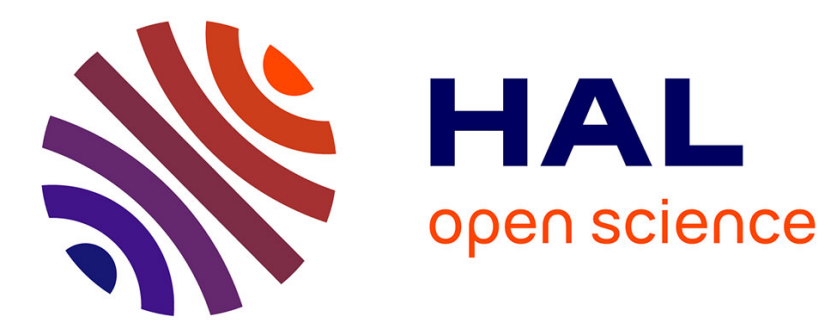

\title{
From stable walking to steering of a 3D bipedal robot with passive point feet
}

Ching-Long Shih, Jessy Grizzle, Christine Chevallereau

\section{To cite this version:}

Ching-Long Shih, Jessy Grizzle, Christine Chevallereau. From stable walking to steering of a 3D bipedal robot with passive point feet. Robotica, 2012, 30 (7), pp.1119-1130. 10.1017/S026357471100138X . hal-00794633

\section{HAL Id: hal-00794633 \\ https://hal.science/hal-00794633}

Submitted on 26 Feb 2013

HAL is a multi-disciplinary open access archive for the deposit and dissemination of scientific research documents, whether they are published or not. The documents may come from teaching and research institutions in France or abroad, or from public or private research centers.
L'archive ouverte pluridisciplinaire HAL, est destinée au dépôt et à la diffusion de documents scientifiques de niveau recherche, publiés ou non, émanant des établissements d'enseignement et de recherche français ou étrangers, des laboratoires publics ou privés. 


\section{From stable walking to steering of a 3D bipedal robot with passive point feet}

Ching-Long Shih ${ }^{+*}$, J. W. Grizzle ${ }^{++}$, and Christine Chevallereau ${ }^{+++}$

+* Department of Electrical Engineering, National Taiwan University of Science and Technology, Taipei, Taiwan. (email: shihcl@mail.ntust.edu.tw)

${ }^{++}$Department of Electrical Engineering and Computer Science, University of Michigan, Ann Arbor, MI USA. (email: grizzle@eecs.umich.edu)

${ }^{+++}$IRCCyN, CNRS, Ecole centrale de Nantes, Nantes, France.

(email :Christine.Chevallereau@irccyn.ec-nantes.fr) 


\begin{abstract}
This paper exploits a natural symmetry present in a $3 \mathrm{D}$ robot in order to achieve asymptotically stable steering. The robot under study is composed of 5-links and unactuated point feet; it has 9 DoF (degree-of-freedom) in the single support phase and six actuators. The control design begins with a hybrid feedback controller that stabilizes a straight-line walking gait for the 3D bipedal robot. The closed-loop system (i.e., robot plus controller) is shown to be equivariant under yaw rotations, and this property is used to construct a modification of the controller that has a local, but uniform, input-to-state stability (ISS) property, where the input is the desired turning direction. The resulting controller is capable of adjusting the net yaw rotation of the robot over a step in order to steer the robot along paths with mild curvature. An interesting feature of this work is that one is able to control the robot's motion along a curved path using only a single predefined periodic motion.
\end{abstract}

KEYWORD:3D underactuated bipedal robot; hybrid zero dynamics; orbital stability; Poincaré map;, steering; stride-to-stride control. 


\section{Introduction}

Research on bipedal walking can be roughly divided along the degree of actuation throughout the gait (full actuation versus underactuation), and whether walking motions are along a straight line or turning is considered. The primary objective of this paper is to study turning motions in underactuated 3D bipedal robots. In addition to the reduced number of actuators, the interest of studying underactuated robots is that the feedback control solution must exploit the robot's natural dynamics in order to achieve balance while walking. In a previous paper ${ }^{1}$, we addressed the control of a 3D bipedal robot with point feet, where the ground contact inhibited yaw motion, but pitch and roll were unconstrained and unactuated. Such contact conditions arise naturally as the limiting case when the surface area of the support foot tends to zero. The first objective of this paper is to remove the restriction on yaw and allow a completely unconstrained and unactuated 3D point foot contact model. The second and primary objective of the paper is to present an event-based controller that steers the robot along paths of low curvature. A novel feature of the solution is that steering is achieved on the basis of a single, predefined, periodic motion corresponding to walking along a straight line.

The ability to turn is an essential feature for stepping around obstacles on a given surface. Honda's ASIMO has demonstrated the important ability to walk forward, 
backward, right, left, up and down stairs, and on uneven terrain ${ }^{2}$. Most of the works that have addressed turning are for bipeds with actuated feet ${ }^{3-7}$. A diverse set of methods for turning has been explored. For instance, by adjusting the swing leg center of mass and hip position trajectories in a trial and error fashion, it is possible to maintain the robot's stability during turning ${ }^{3}$. Generating a turning motion of a bipedal robot by slipping the feet on the ground was presented ${ }^{4}$. To generate the slip motion, the authors predict the amount of slip using the hypothesis that the turning motion is caused by the effect of minimizing the power generated by floor friction. It has been shown that straight line and turning walks could be realized by nonlinear oscillator systems, and the turning motion leads to the change of the duty ratios of the legs ${ }^{5}$. Biped turning motions with ZMP-based footstep planning were studied ${ }^{6,7}$.

The authors ${ }^{8-11}$ have developed an elegant and rigorous setting for stable walking and steering of fully actuated $3 \mathrm{D}$ robots on the basis of geometric reduction and passivity-based control. The controlled geometric reduction decouples the biped's sagittal-plane motion from the yaw and lean modes. Passivity-based control is used to create and stabilize planar limit cycles that arise from the sagittal component of the reduction. Steering is achieved by adjusting the yaw set point of the within-stride passivity-based controller.

We study here a 3D passive point contact at the leg end, and, for a 5-link robot, seek 
a time-invariant feedback controller that creates an exponentially stable, periodic walking motion, along with the ability to steer the yaw orientation of the robot with respect to an inertial frame, that is, the robot's direction of travel. In our previous studies on 3D bipedal robots, we assumed a model where the ground contact inhibited yaw motion, but pitch and roll were unconstrained and unactuated. Starting with a simple 3-link model ${ }^{12}$ and followed by a 5 -link model ${ }^{1}$, we used the techniques of virtual constraints, hybrid zero dynamics and event-based control to achieve exponentially stable, periodic walking motions ${ }^{13}$. In the present study we extend these results to design and stabilize periodic orbits for a 3D bipedal robot with point feet modeled as a passive three degree of freedom pivot.

The control approach presented in this paper allows us to change the direction of motion of the robot through the net yaw motion about the stance foot over a step. An event-based feedback controller distributes set point commands to the actuated joints in order to achieve a desired amount of turning, as opposed to the continuous corrections used ${ }^{9}$. The key property that allows this to work is based on a natural symmetry of the hybrid robot model that was identified ${ }^{14}$. 


\section{Model}

\subsection{Description of the Robot}

The 3D bipedal robot discussed in this work is shown in Fig. 1. It consists of five links: a torso and two legs with knees that are terminated with "point-feet." Each hip consists of a revolute joint with 2 degrees of freedom and each knee is formed by a 1 degree of freedom revolute joint; these six joints $\left(q_{3}, q_{4}, \cdots, q_{8}\right)$ (three in each leg) are independently actuated. The stance leg end is assumed to act as a passive pivot, so the leg end is modeled as a point contact with 3 degrees of freedom $\left(q_{0}, q_{1}, q_{2}\right)$ and no actuation. In total, the biped in the single support phase has 9 degrees of freedom, and there are hence 3 degrees of underactuation. The coordinate $q_{0}$ gives the absolute orientation of the biped with respect to the world frame. This variable will be called yaw in what follows.

Assuming support on leg 1 , a set of generalized coordinates $q=\left[q_{0}, q_{1}, \cdots, q_{8}\right]^{T}$ is defined as shown in Fig. 1. The coordinates $\left(q_{0}, q_{1}, q_{2}\right)$ are unactuated (passive contact), while $\left(q_{3}, q_{4}, \cdots, q_{8}\right)$ are independently actuated (active joints). The position of the robot with respect to an inertial frame is defined by adding three variables $\left(x_{1}, y_{1}, z_{1}\right)$, which are the Cartesian coordinates of the stance foot and are constant during each single support phase. When leg 2 is the stance leg, then a new set of generalized coordinates $q$ is defined as shown in Fig. 2, and the same notation is 
employed as when leg 1 is the supporting leg.

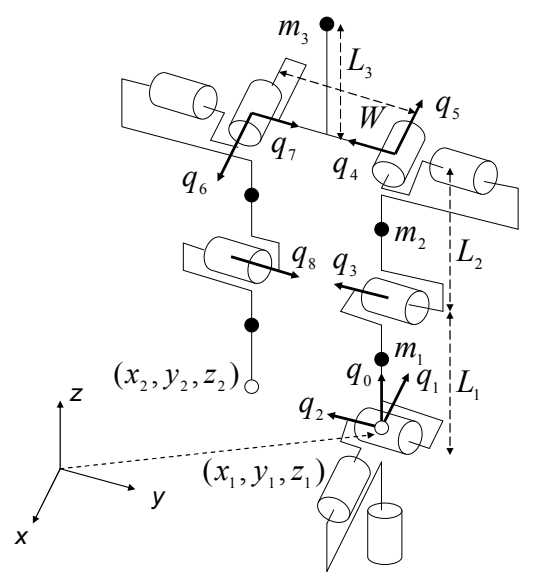

Fig. 1. A 3D point-feet bipedal robot when support on leg 1, the 3 degrees of freedom at the leg end are unactuated. For simplicity, each link is modeled by a point mass at its center.

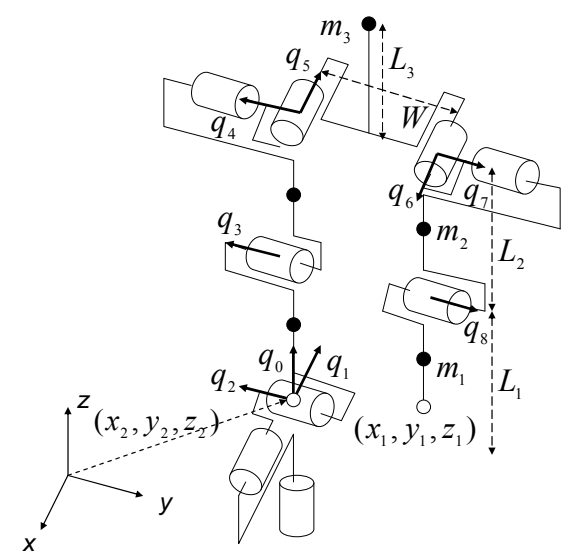

Fig. 2. The generalized coordinates of the bipedal robot when support on leg 2 .

\subsection{Dynamic Model and the Walking Gait}

A bipedal walking gait consists of a single support phase and a double support phase.

The dynamic model for the robot in the single support phase on leg 1 is represented as 


$$
D(q) \ddot{q}+C(q, \dot{q}) \dot{q}+N(q)=B u=\left[\begin{array}{c}
0_{3 \times 6} \\
I_{6 \times 6}
\end{array}\right] u,
$$

where $D(q)$ is the positive-definite $9 \times 9$ mass-inertia matrix, $C(q, \dot{q})$ is the $9 \times 9$ Coriolis matrix, $N(q)$ is the $9 \times 1$ gravity vector, $B$ is an $9 \times 6$ full-rank, constant matrix indicating whether a joint is actuated or not, and $u$ is the $6 \times 1$ vector of input torques. The models for support on leg 2 can be written in a similar way by using a hip width of $-W$ in place of $W$.

During the double support phase, the biped's configuration variables do not change, but velocities undergo a jump. The double support phase is assumed to be instantaneous, and to consist of two distinct subphases: the impact and coordinate relabeling. Analogously to Chevallereau et al. ${ }^{1}$, the overall impact model is written as

$$
q^{+}=\Delta_{q}\left(q^{-}\right)
$$

and

$$
\dot{q}^{+}=\Delta_{\dot{q}}\left(q^{-}, \dot{q}^{-}\right)
$$

where $\left(q^{-}, \dot{q}^{-}\right)$are joint angles and joint velocities of the bipedal robot for support on leg 1 just before the impact; and $\left(q^{+}, \dot{q}^{+}\right)$are joint angles and joint velocities of the bipedal robot for support on leg 2 and immediately after the impact. The calculation of $\left(q^{+}, \dot{q}^{+}\right)$includes the change of coordinates for the transfer of support onto leg 1 from 2. The transformation from one set of coordinates at the end of a step to the other set of coordinates is done as follows. Compute the orientation and the angular 
velocity of the swing leg shin. From this, one deduces $\left(q_{0}, q_{1}, q_{2}\right)$ that are compatible with this orientation; and then, one deduces $\left(\dot{q}_{0}, \dot{q}_{1}, \dot{q}_{2}\right)$ yielding the angular velocity of the swing shin. The angles $\left(q_{3}, q_{4}, \cdots, q_{8}\right)$ exchange their role viz $\left(q_{8}, q_{7}, \cdots, q_{3}\right)$.

Define state variables $x_{j}=\left[\begin{array}{c}q \\ \dot{q}\end{array}\right]$, and let $x_{j}^{+}=\left[\begin{array}{c}q^{+} \\ \dot{q}^{+}\end{array}\right]$and $x_{j}^{-}=\left[\begin{array}{c}q^{-} \\ \dot{q}^{-}\end{array}\right]$, where the subscript $j \in\{1,2\}$ denotes the stance leg number. Then a complete walking motion of the robot can be expressed as a nonlinear system with impulse effects and written as

$$
\Sigma:\left\{\begin{array}{cc}
\dot{x}_{1}=f_{1}\left(x_{1}\right)+g_{1}\left(x_{1}\right) u_{1} & x_{1}^{-} \notin S_{1} \\
x_{2}^{+}=\Delta_{1}\left(x_{1}^{-}\right) & x_{1}^{-} \in S_{1} \\
\dot{x}_{2}=f_{2}\left(x_{2}\right)+g_{2}\left(x_{2}\right) u_{2} & x_{2}^{-} \notin S_{2} \\
x_{1}^{+}=\Delta_{2}\left(x_{2}^{-}\right) & x_{2}^{-} \in S_{2}
\end{array},\right.
$$

where $S_{1}=\left\{(q, \dot{q}) \mid z_{2}(q)=0, \dot{z}_{2}(q)<0\right\}$ is the switching surface, $u_{1}=u$, and

$$
\begin{gathered}
f_{1}\left(x_{1}\right)=\left[\begin{array}{c}
\dot{q} \\
D^{-1}(q)(-C(q, \dot{q}) \dot{q}-N(q))
\end{array}\right] \\
g_{1}\left(x_{1}\right)=\left[\begin{array}{c}
0 \\
D^{-1}(q) B
\end{array}\right], \\
x_{2}^{+}=\Delta_{1}\left(x_{1}^{-}\right)=\left[\begin{array}{c}
\Delta_{q}\left(q^{-}\right) \\
\Delta_{\dot{q}}\left(q^{-}, \dot{q}^{-}\right)
\end{array}\right] .
\end{gathered}
$$

When leg 2 is the support leg, the same derivation produces

$$
\begin{gathered}
S_{2}=\left\{(q, \dot{q}) \mid z_{1}(q)=0, \dot{z}_{1}(q)<0\right\}, u_{2}, f_{2}\left(x_{2}\right), g_{2}\left(x_{2}\right) \text { and } \\
x_{1}^{+}=\Delta_{2}\left(x_{2}^{-}\right) .
\end{gathered}
$$




\subsection{Nominal Motion of Walking Along a Straight Line}

A solution $x_{1}(t), 0 \leq t \leq T_{1}$ and $x_{2}(t), 0 \leq t \leq T_{2}$ of the model (4) for inputs $u_{1}(t)$ and $u_{2}(t)$ is periodic with period $T_{1}+T_{2}$ if $x_{2}^{+}(0)=\Delta_{1}\left(x_{1}^{-}\left(T_{1}\right)\right)$ and $x_{1}^{+}(0)=\Delta_{2}\left(x_{2}^{-}\left(T_{2}\right)\right)$. A periodic solution is said to be a symmetric gait along the $x$-axis of the world frame if the duration of each step is equal, that is $T_{1}=T_{2}=T$ for some $T>0$, and for all $0 \leq t \leq T$

$$
x_{1}(t)=E x_{2}(t),
$$

where

$$
E=\left[\begin{array}{cc}
S & 0_{9 \times 9} \\
0_{9 \times 9} & S
\end{array}\right]
$$

and

$$
S=\operatorname{diag}\{-1,-1,1,1,1,-1,-1,1,1\} .
$$

Remark 1: If the condition (7) holds except for anti-symmetry of the yaw orientation $q_{0}(t)$ of the left and right legs, more precisely, if the condition (7) becomes $x_{1}(t)=E x_{2}(t)+f$ where $f$ has only its first component different from zero, then the gait is still symmetric and corresponds to periodic walking along a straight line other than the x-axis of the world frame; indeed, the direction of motion is at an angle $-f_{1} / 2$ with respect to the $\mathrm{x}$-axis. 


\subsection{An Invariance Property of the Model}

The yaw-symmetry defined here is a special case of the invariance under $\mathrm{SO}(3)^{14}$.

Let $G$ denote the group of rotations about the z-axis of the world frame, which can be identified with the circle, or $[-\pi, \pi)$. This induces an action on the configuration space $Q$ by $\Phi: G \times Q \rightarrow Q$ where

$$
\Phi_{g}(q)=\left(q_{0}+g, q_{1}, \ldots, q_{8}\right)
$$

This in turn lifts to an action on the state space $T Q$ by $\Psi_{g}(q, \dot{q})=T \Phi_{g}(\dot{q}, q)=\left(\Phi_{g}(q), \dot{q}\right)$. A function $\varphi: T Q \rightarrow R^{k}, k \geq 1$, is invariant under $G$ if for all $g \in G$ and $(q, \dot{q}) \in T Q, \quad \varphi \circ\left(\Phi_{g}(q), \dot{q}\right)=\varphi(q, \dot{q})$; and $\Gamma: T Q \rightarrow T Q$ is equivariant under $G$ if for all $g \in G$ and $(q, \dot{q}) \in T Q$, $\Gamma \circ \Psi_{g}(q, \dot{q})=\Psi_{g} \circ \Gamma(q, \dot{q})$

Proposition 0: For all $g \in G, \Psi_{g}: S_{1} \rightarrow S_{1}$ and $\Psi_{g}: S_{2} \rightarrow S_{2}$.

Proof:

$z_{1}(q)$ and $z_{2}(q)$ are the heights of leg-1 and leg-2 above the ground, respectively, and hence are invariant under yaw rotations. It follows that $S_{1}$ and $S_{2}$ are invariant sets as well. Q.E.D

Proposition 1: Let $u_{1}(q, \dot{q})$ and $u_{2}(q, \dot{q})$ be locally Lipschitz continuous state 
variable feedbacks and let $x_{t}\left(x_{0}\right)$ denote a solution of the resulting closed-loop hybrid model (4) with initial condition $x_{0}$. If $u_{1}$ and $u_{2}$ are invariant under $G$, then $x_{t}(\cdot)$ is equivariant under $G$.

Proof:

In Spong and Bullo ${ }^{14}$, it is shown that the kinetic energy term of the Lagrangian model and the impact surfaces are invariant under $S O(3)$, the group of rotations of the world frame, and the impact maps are equivariant under $S O(3)$. Hence, these are in particular invariant and equivariant respectively under $G$ the group of rotations about the z-axis. Because the z-axis of the world frame is aligned with the direction of gravity, the potential energy is invariant under $G$. From this and the hypothesis on the feedbacks, the vector fields of the closed-loop system are equivariant under $G$. Putting all of this together, the solutions of the closed-loop system are equivariant.

In words, the proposition analyzes the situation where the within-stride feedback controller does not depend on the yaw orientation of the robot (i.e., rotations with respect to the $\mathrm{z}$-axis). In this case, the following two motions will result in the robot having the same final pose: (a) the robot is initialized from a given pose and advances for $T$ units of time in single support and its state is then rotated by $g$ radians about the z-axis; (b) the initial pose is first rotated by $g$ radians about the z-axis and then the 
robot walks for $T$ units of time. The state considered here does not include the three Cartesian variables $\left(x_{1}, y_{1}, z_{1}\right)$ or $\left(x_{2}, y_{2}, z_{2}\right)$ describing the absolute position of the robot. It includes only the angular variables $\left(q_{0}, \cdots, q_{8}\right)$. This proposition will have a consequence on stability and on the possibility to steer the robot as examined later in Section 5 .

\section{Gait and Within-Stride Controller Design}

The nominal gait and controller designs proceed as in Chevallereau et al. ${ }^{1}$ and only the key points are summarized here. The new contributions are given in Propositions 2-4 which state properties of the controller and closed-loop system that will be of great help in designing a steering controller.

\subsection{Virtual Constraints and Within-Stride Controller}

A direct form of the constraint is used

$$
y_{6 \times 1}=h(q)=q_{a}-h_{d}(\theta),
$$

where $q_{a}=\left[q_{3}, q_{4}, \cdots, q_{8}\right]^{T}$ is the vector of actuated coordinates, $\theta=\theta(q)$ is a quantity that is strictly monotonic along a typical walking gait, and $h_{d}(\theta)$ is the desired evolution of the actuated variables as a function of $\theta$. When the shin and thigh have the same length, the angle of the virtual leg is $\theta=-q_{2}-q_{3} / 2$. The input-output linearizing controller 


$$
u=u^{*}-\left(\frac{\partial h}{\partial q} D^{-1} B\right)^{-1}\left(\frac{K_{p}}{\varepsilon^{2}} y+\frac{K_{d}}{\varepsilon} \dot{y}\right)
$$

with

$$
u^{*}=\left(\frac{\partial h(q)}{\partial q} D^{-1} B\right)^{-1}\left(\frac{\partial^{2} h_{d}(\theta)}{\partial \theta^{2}} \dot{\theta}^{2}(t)+\frac{\partial h(q)}{\partial q} D^{-1}(C(q, \dot{q}) \dot{q}+N(q))\right)
$$

and appropriate choices for the gains $K_{p}, K_{d}$, and $\varepsilon$ will allow the errors in the virtual constraints to be driven asymptotically to zero.

Proposition 2: Because the virtual constraints in (11) are invariant under $G$, the input-output linearizing feedback $u$ in (12) is also invariant under $G$. If $h_{d}(\theta)$ is twice differentiable and the second derivative is Lipschitz continuous, then $u$ is Lipschitz continuous.

Proof: This is immediate from the expressions for the controller in (12) and (13).

\subsection{Poincaré Map of the Full-model}

Consider the hybrid model (4) in closed-loop with the feedback (12). The flow map $x$ is the (partial) map defined by taking an initial condition in $S_{1}$, applying the impact $x_{2}^{+}=\Delta_{1}\left(x_{1}^{-}\right)$and following the evolution of the Euler-Lagrange equations until $x_{2}(t) \in S_{1}$; the flow map $P_{21}: S_{2} \rightarrow S_{1}$ is defined similarly. The Poincaré map $P: S_{2} \rightarrow S_{2}$ is the composition of the two flow maps, $P=P_{21} \circ P_{12}$. The map 
$P_{1}: S_{1} \rightarrow S_{1}$ by $P_{1}=P_{12} \circ P_{21}$ is similarly defined. It is diffeomorphic to $P$ and hence the choice of one versus the other is arbitrary.

A fixed point is defined by $x_{2}^{*}=P\left(x_{2}^{*}\right)$ and corresponds to a periodic walking motion. The fixed point corresponds to a symmetric gait aligned along the x-axis of the world frame if $x_{1}^{*}=P_{21}\left(x_{2}^{*}\right)$ satisfies $x_{1}^{*}=E x_{2}^{*}$, for $E$ defined in (8). The Poincaré map gives rise to a discrete-time system

$$
x_{2}(k+1)=P\left(x_{2}(k)\right)
$$

evolving on the switching surface $S_{2}$, where $x_{2}$ are the state variables.

Proposition 3: Under the hypotheses of Propositions 1 and 2, the Poincaré map is equivariant under the action of $G$, the group of rotations about the z-axis of the world frame. In particular, for each $g \in G$ and $x \in S_{2}$,

$$
P \circ \Psi_{g}(x)=\Psi_{g} \circ P(x),
$$

and hence if $x^{*}$ is a fixed point of $P$, so is $\Psi_{g}\left(x^{*}\right)$ for every $g \in G$.

Proof:

The proof is almost immediate from Proposition 1. The Poincaré map is computed by sampling the solution of the model when the swing leg impacts with the ground ${ }^{13}$. From Proposition 1, the solution is equivariant under $G$. As noted in the Proposition 
0 , the impact surface is invariant under $G$, and hence the time-to-impact map is invariant under $G$. These two facts together prove the proposition. Q.E.D

It follows that if the within-stride feedback controller is independent of $q_{0}$, periodic orbits cannot be asymptotically stable. Asymptotically stable equilibrium points must be isolated; however, Proposition 2 shows that equilibrium points cannot be isolated as they belong to a one-parameter family of equilibrium points corresponding to rotations about the z-axis. At best, they can be asymptotically stable "modulo $G$ ". This could be formalized by defining the quotient of the closed-loop hybrid model by $G$, but this will not be pursued here.

The linearization of (14) about a fixed-point $x_{2}^{*}$ gives rise to a linearized system,

$$
\delta x_{2}(k+1)=A \delta x_{2}(k)
$$

where

$$
A=\left[\begin{array}{lllll}
A_{0} & A_{1} & A_{3} & \cdots & A_{17}
\end{array}\right]_{17 \times 17}
$$

is the Jacobian of the Poincaré map $P$. The lack of asymptotic stability manifests itself in the linearization of the Poincaré map as follows.

Proposition 4: Under the hypotheses of Propositions 1 and 2, the first column of $A$ is given by

$$
A_{0}=\left[\begin{array}{llll}
1 & 0 & \ldots & 0
\end{array}\right]^{T}
$$


and hence $A$ always has an eigenvalue at 1.0. Recall that the exponential stability of a fixed-point is equivalent to the eigenvalues of $A$ having magnitude strictly less than one $e^{13}$.

\subsection{The Restricted Poincaré Map}

Following the method used in Chevallereau et al. ${ }^{1}$, it can be shown that in $S_{2} \cap Z$ the state of the robot can be represented using only five independent variables $x^{z}=\left[q_{0}, q_{1}, \dot{q}_{0}, \dot{q}_{1}, \dot{\theta}\right]^{T}$, where $Z=\left\{(q, \dot{q}): y_{c}(q)=0, \dot{y}_{c}(q, \dot{q})=0\right\}$, if the output for the feedback control design is modified as

$$
y_{c}=h\left(q, y_{i}, \dot{y}_{i}\right)=q_{a}-h_{d}(\theta)-h_{c}\left(\theta, y_{i}, \dot{y}_{i}\right) \text {. }
$$

The correction term $h_{c}$ is taken to be a three-times continuously differentiable function of $\theta$,

$$
\left\{\begin{array}{c}
h_{c}\left(\theta_{i}, y_{i}, \dot{y}_{i}\right)=y_{i} \\
\frac{\partial}{\partial \theta} h_{c}\left(\theta_{i}, y_{i}, \dot{y}_{i}\right)=\frac{\dot{y}_{i}}{\dot{\theta}_{i}} \\
h_{c}\left(\theta, y_{i}, \dot{y}_{i}\right)=0, \quad 0.5 \theta_{i}+0.5 \theta_{f} \leq \theta \leq \theta_{f}
\end{array}\right.
$$

where $y_{i}$ and $\theta_{i}$ are the initial value of output $y$ and $P_{12}$ for the current step, and $\theta_{f}$ is the final value of $\theta$ for the current step. The restricted map $P^{z}: S_{2} \cap Z \rightarrow S_{2} \cap Z$, induces a discrete-time system

$$
x_{k+1}^{z}=P^{z}\left(x_{k}^{z}\right) .
$$

Defining $\delta x_{k}^{z}=x_{k}^{z}-x^{z^{*}}$, the restricted map linearized about a fixed point $x^{z^{*}}$, 
$x^{z^{*}}=\left[q_{0}^{-}, q_{1}^{-}, \dot{q}_{0}^{-}, \dot{q}_{1}^{-}, \dot{\theta}^{-}\right]^{T}$, gives rise to a linearized system

$$
\delta x_{k+1}^{z}=A^{z} \delta x_{k}^{z}
$$

Remark 2: It can be easily shown that the restricted map has the same $G$ equivariance properties as the full map.

\section{Nominal Stable Walking Along a Straight Line}

The physical parameters of the $3 \mathrm{D}$ biped used in this study were chosen as in Table

I. The parameters result in the center of gravity of the biped being located below the midpoint of the hips.

\section{TABLE I}

PARAMETERS FOR THE 3D BIPEDAL ROBOT (in MKS)

\begin{tabular}{|c|c|c|c|c|c|c|}
\hline$W$ & $L_{1}$ & $L_{2}$ & $L_{3}$ & $m_{1}$ & $m_{2}$ & $m_{3}$ \\
\hline 0.15 & 0.275 & 0.275 & 0.10 & 0.875 & 0.875 & 5.5 \\
\hline
\end{tabular}

\subsection{A Periodic Motion}

A nominal periodic walking motion corresponding to a symmetric gait along the $\mathrm{x}$-axis of the world frame is used. An optimal state $x_{2}^{*}=\left(q^{-}, \dot{q}^{-}\right)$that minimize a given cost criterion, such as energy consumed per step length, is found ${ }^{1.13}$. The search procedure is carried out in MATLAB with the FMINCON function of the 
optimization toolbox. For these parameters, a periodic orbit was obtained and defined by $x_{2}^{*}=\left(q^{-}, \dot{q}^{-}\right)$below

$$
\begin{aligned}
& x_{2}^{*}=\left[\begin{array}{llllll}
0.3151, & -0.0838, & -0.1317, & 0.0997, & -0.7543, & 0.1948,
\end{array}\right. \\
& \begin{array}{llllll}
-0.0592, & -1.0471, & 0.1809, & -0.1260, & 0.2088, & -0.8899,
\end{array} \\
& \text { 0.3175, } 0.0449, \quad 0.5374, \quad-1.5619, \quad 0.9187, \quad 0.6288]^{\prime} .
\end{aligned}
$$

Stick diagrams for the first step of the periodic walking gaits are presented in Fig. 3.

The walking cycle has a period of $T=0.4477$ seconds, a step size of $L=0.0976 \mathrm{~m}$, and an average walking speed of $0.218 \mathrm{~m} / \mathrm{sec}$ (or 0.396 body lengths per second). The nominal gait's joint profiles and angular velocities over two consecutive steps are shown in Fig. 4.

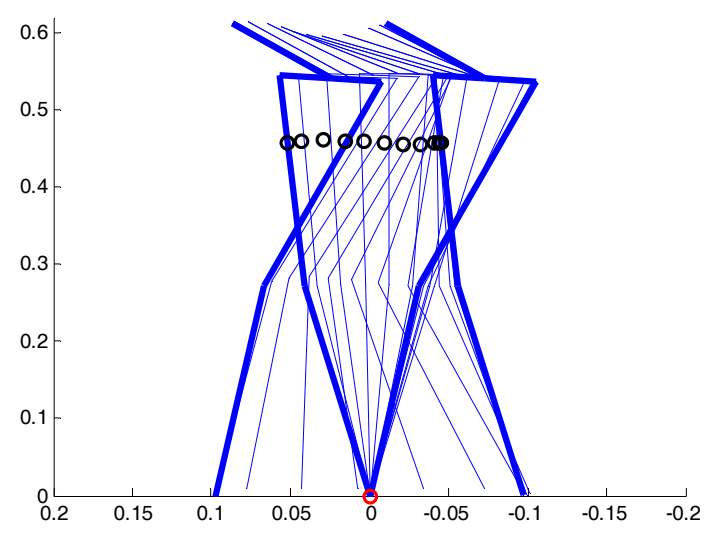

(a) x-z plane (unit:m) 


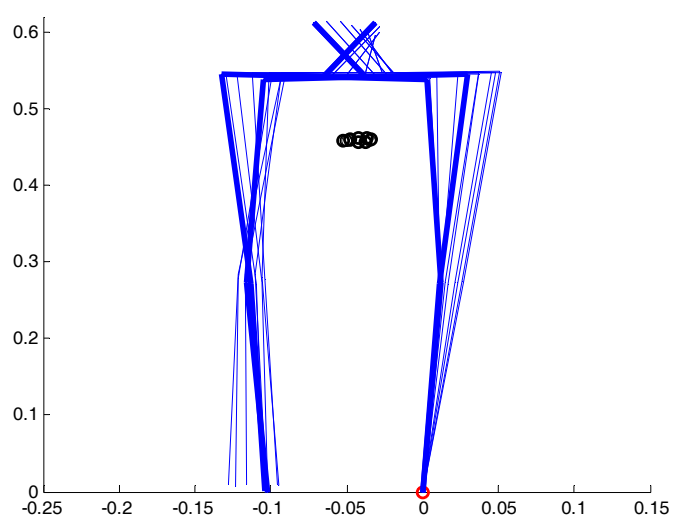

(b) y-z plane (unit:m)

Fig. 3. Stick diagrams for the first step of the periodic walking gait.
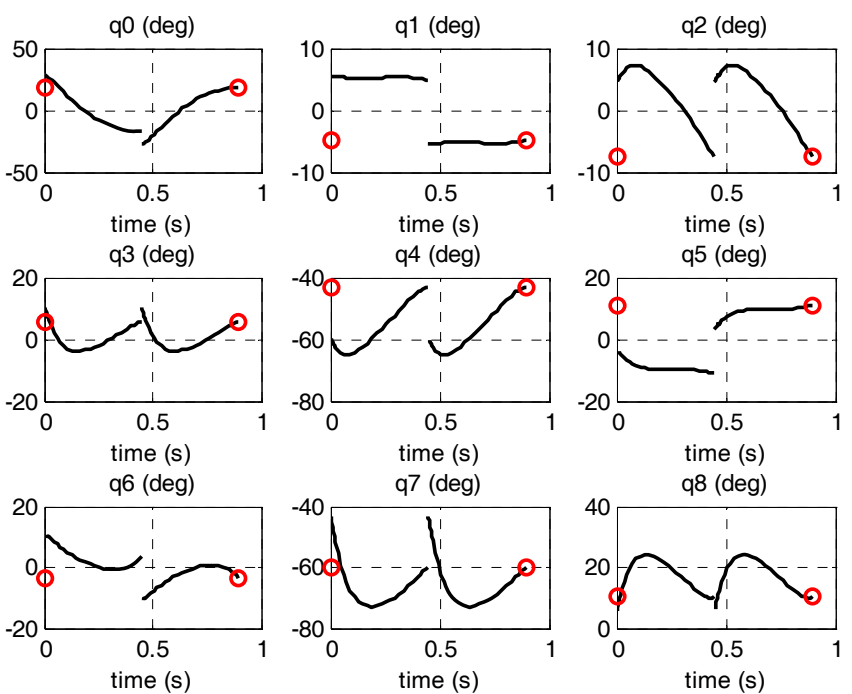

Fig. 4. Joint profiles of the obtained periodic motion over two steps, where the small circles represent $q^{-}$. Joint angles $q_{i}, \mathrm{i}=0,1, \ldots, 8$, are shown in the first half in which leg 1 on support, and joint angles $q_{i}, \mathrm{i}=0,1, \ldots, 8$, are shown on the second half in which leg 2 on support. 


\subsection{Stability Analysis}

First, the control law (12) for the full model of the 3D biped was used with virtual constraints $y=q_{a}-h_{d}(\theta)-h_{c}\left(\theta, y_{i}, \dot{y}_{i}\right)$; the PD control gains used are $K_{p}=50.0$, $K_{d}=10.0$ and $\varepsilon=0.1$. To test the stability of this control law around the periodic motion, the eigenvalues of the restricted Poincaré map are numerically estimated with $\Delta q_{i}=0.0750^{\circ}, \Delta \dot{q}_{i}=0.3750^{\circ} \mathrm{s}^{-1}$. The linearization of the Poincare map $A$ and $A^{z}$ were computed, and their eigenvalues are shown in Table II and Table III, respectively, where only the 8 largest eigenvalues of $A$ are shown.

TABLE II

EIGENVALUES OF Poincaré MAP $A$

\begin{tabular}{|c|c|c|}
\hline$i$ & $\lambda_{i}$ & $\left|\lambda_{i}\right|$ \\
\hline 1 & 1 & 1 \\
\hline 2 & 0.7733 & 0.7733 \\
\hline 3 & $-0.4492+j 0.6287$ & 0.7727 \\
\hline 4 & $-0.4492-j 0.6287$ & 0.7727 \\
\hline 5 & 0.3071 & 0.3071 \\
\hline 6 & 0.0063 & 0.0063 \\
\hline 7 & 0.0014 & 0.0014 \\
\hline 8 & 0.0001 & 0.0001 \\
\hline
\end{tabular}


TABLE III

EIGENVALUES OF HZD RESTRICTED Poincaré MAP $A^{z}$

\begin{tabular}{|c|c|c|}
\hline$i$ & $\lambda_{i}$ & $\left|\lambda_{i}\right|$ \\
\hline 1 & 1 & 1 \\
\hline 2 & 0.7873 & 0.7873 \\
\hline 3 & $-0.4415+j 0.5949$ & 0.7408 \\
\hline 4 & $-0.4415-j 0.5949$ & 0.7408 \\
\hline 5 & 0.3225 & 0.3225 \\
\hline
\end{tabular}

To illustrate the orbit's local stability of the fixed-point $x_{2}^{*}$, under the continuous controller, a perturbation of $\pi / 6$ is added to the initial value of $q_{0}$ and very small initial errors are introduced on other joint angles. All joint velocities are also perturbed by very small amounts. The use of small perturbations is due to the fact that the region of attraction is relatively small. Fig. 5 shows the evolution of the final values of the uncontrolled variables $\left(q_{0}, q_{1}, q_{2}\right)$ at each step. These variables converge slowly to their desired values except that $q_{0}$ moves to an offset value different from the desired one. Fig. 6 shows phase-plane plots of $\left(q_{0}, q_{1}, q_{2}, \theta\right)$. The convergence toward a periodic motion is clear for each variable. Note that the value of $q_{0}$ does not change signs from one step to the next; therefore, the robot is following a straight line path that is not aligned with the x-axis of the world frame (the path will 
be shown in Fig. 11 for comparison with the case of having an additional stride-to-stride controller).
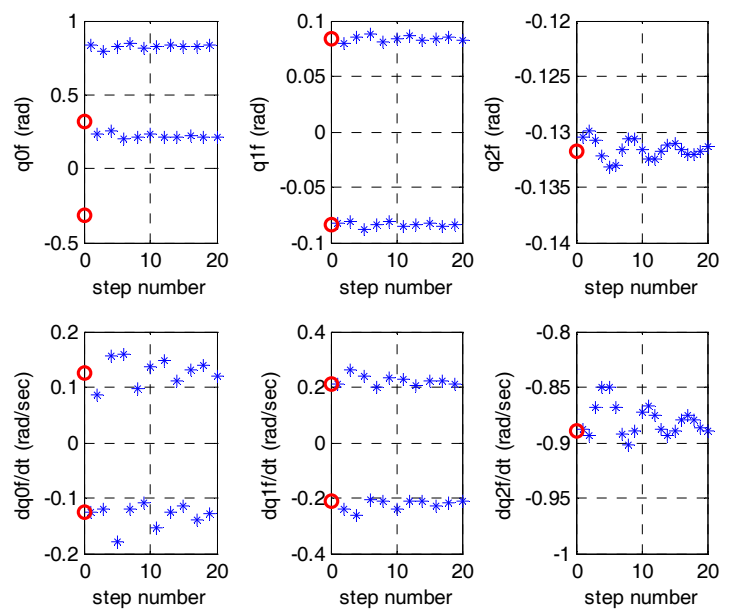

Fig. 5. Evolution of continuous control of unactuated joints $\left(q_{0}, q_{1}, q_{2}\right)$ at the end of each step when a perturbation of $\pi / 6$ is added to $q_{0}$. The small circles represent the values on the desired periodic orbit.
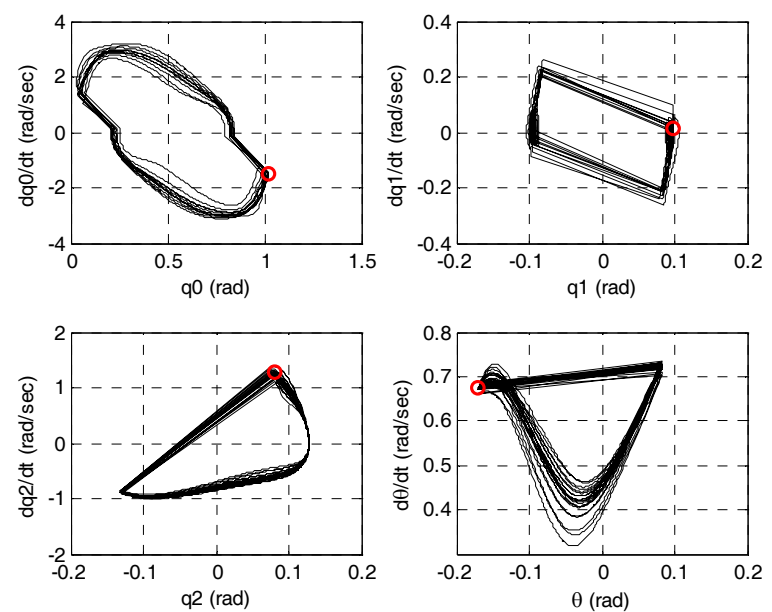

Fig. 6. Phase-plane plots for continuous control $\left(q_{0}, q_{1}, q_{2}, \theta\right)$ when a perturbation of $\pi / 6$ is added on the initial value of $q_{0}$. Each variable converges to periodic motion.

The small circles represent the initial state. 
If a desired periodic gait is not exponentially stable or the region of attraction is too small, then event-based control can be designed and integrated with the continuous, stance-phase controller. The idea is to introduce a vector of parameters that is held constant during the stance phase and updated at each impact. Here, it will be updated on the basis of the state of the hybrid zero dynamics. The output is augmented with an additional term,

$$
y=q_{a}-h_{d}(\theta)-h_{c}\left(\theta, y_{i}, \dot{y}_{i}\right)-h_{s}(\theta, \beta),
$$

in which $h_{s}(\theta, \beta)$ depending on a vector of parameters $\beta \in \mathrm{B}_{0}$, where $\mathrm{B}_{0}$ is an open neighborhood of the origin of $R^{6}$, and where

$$
h(\theta, 0)=0, \quad \theta_{i} \leq \theta \leq \theta_{f}
$$

with

$$
\left\{\begin{array}{c}
h_{s}\left(\theta_{i}, \beta\right)=0 \\
\frac{\partial h_{s}}{\partial \theta}\left(\theta_{i}, \beta\right)=0 \\
h_{s}\left(0.5 \theta_{i}+0.5 \theta_{f}, \beta\right)=\beta \\
h_{s}(\theta, \beta)=0, \quad 0.1 \theta_{i}+0.9 \theta_{f} \leq \theta \leq \theta_{f}
\end{array} .\right.
$$

Specifically, $h_{s}(\theta, \beta)$ is taken to be a fifth-order polynomial for $\theta_{i} \leq \theta \leq 0.1 \theta_{i}+0.9 \theta_{f}$

The restricted Poincaré map can now be viewed as a nonlinear control system on $S_{2} \cap Z$ with input $\beta_{k}$, namely

$$
x_{k+1}^{z}=P^{z}\left(x_{k}^{z}, \beta_{k}\right),
$$


where $\beta_{k}$ is the value of $\beta$ during the step $k$. Linearizing this nonlinear system about the fixed point and the nominal parameter value $\beta^{*}=0_{6 \times 1}$ leads to

$$
\delta x_{k+1}^{z}=A^{z} \delta x_{k}^{z}+F \beta_{k}
$$

where $F$ is the Jacobian of the map $P^{z}$ with respect to $\beta$.

Next, design a feedback matrix

$$
\beta_{k}=-K \delta x_{k}^{z}
$$

such that the eigenvalues of $\left(A^{z}-F K\right)$ have magnitude strictly less than one. This will exponentially stabilize the fixed point. Then a $6 \times 5$ gain matrix $K$ is calculated via discrete linear quadratic regulator (DLQR) theory. The eigenvalues of the linearized map with closed-loop stride-to-stride controller are shown in Table IV. All the eigenvalues have magnitude less than 1.0 , and thus the obtained nominal orbit $x_{2}^{*}$ is locally exponentially stable for $\varepsilon$ in (12) sufficiently small ${ }^{17}$. 
TABLE IV

EIGENVALUES OF STRIDE-TO-STRIDE CONTROL

\begin{tabular}{|c|c|c|}
\hline$i$ & $\lambda_{i}$ & $\left|\lambda_{i}\right|$ \\
\hline 1 & 0.5891 & 0.5891 \\
\hline 2 & $-0.3284+j 0.0778$ & 0.3375 \\
\hline 3 & $-0.3284-j 0.0778$ & 0.3375 \\
\hline 4 & 0.2026 & 0.2026 \\
\hline 5 & 0.0688 & 0.0688 \\
\hline
\end{tabular}

To illustrate the orbit's local stability at the fixed-point $x_{2}^{*}$, an initial error of $-1^{\circ}$ is introduced on each joint and a velocity error of $-3^{\circ} s^{-1}$ is introduced on each joint velocity. Fig. 7 shows phase-plane plots of $\left(q_{0}, q_{1}, q_{2}, \theta\right)$. The convergence toward a periodic motion is clear for these variables. Fig. 8 shows evolution of the center of mass in the $x-y$ plane, for the 3D biped's full model under closed-loop walking control, with the initial condition perturbed from $x_{2}^{*}$. The value of $q_{0}$ is symmetric from one step to the next; therefore, the robot is following a straight line along the $\mathrm{x}$-axis of the world frame. 

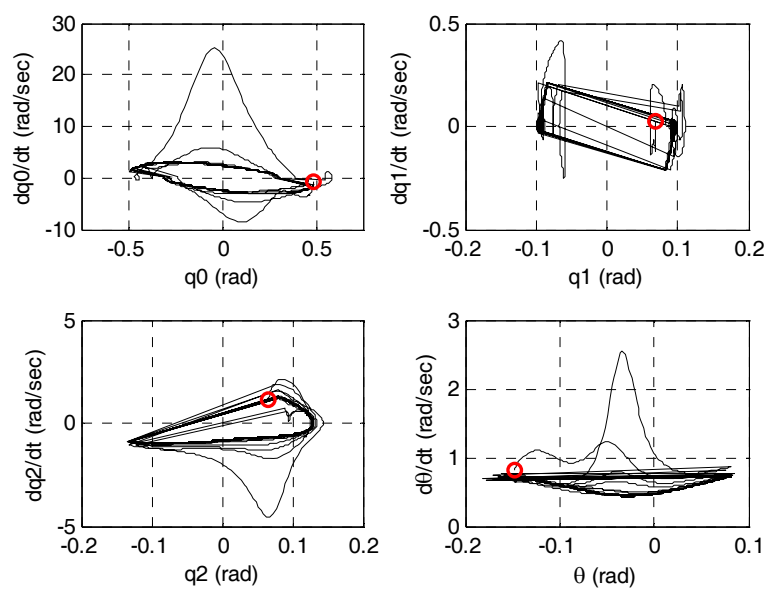

Fig. 7. Phase-plane plots for $\left(q_{0}, q_{1}, q_{2}, \theta\right)$. The small circles represent the initial state. Each variable converges to a periodic motion.

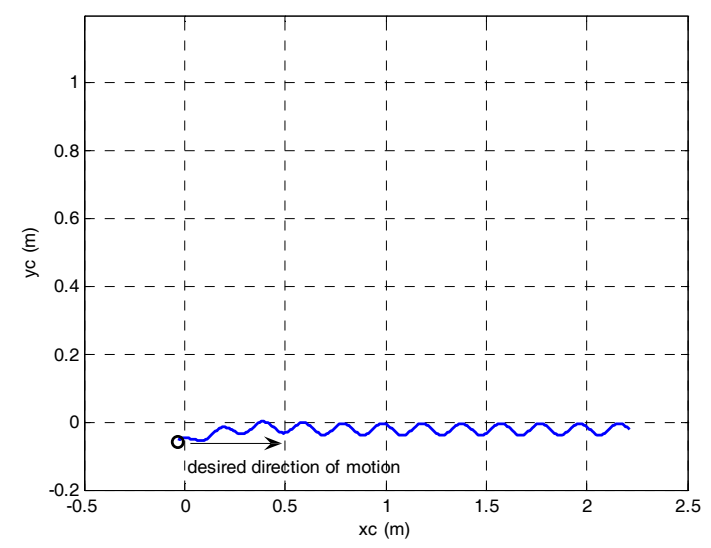

Fig. 8. Evolution of the center of mass in the $x-y$ plane, for the 3D biped's full model under closed-loop walking control, with the initial condition perturbed from $x_{2}^{*}$, where the small circle denotes the starting position.

With the stride-to-stride controller, there is no longer an eigenvalue with magnitude one, meaning that the closed-loop system is no longer invariant under rotations by $q_{0}$. 
In particular, the asymptotic value of $q_{0}$ will return to the fixed point if an initial error is introduced, which was not the case without the feedback gain $K$. For instance, when a perturbation of $\pi / 6$ is added to the initial value of $q_{0}, q_{0}$ converges to the desired value quickly. Fig. 9 shows the evolution of the final values of the uncontrolled variables $\left(q_{0}, q_{1}, q_{2}\right)$ from one step to the next. These variables converge quickly toward the periodic motion. Fig. 10 shows phase-plane plots of $\left(q_{0}, q_{1}, q_{2}, \theta\right)$. The convergence toward the periodic motion is also clear for these variables. Fig. 11 shows the evolution of the center of mass in the $x-y$ plane; the robot returns within $2 \%$ of the desired direction after 3 steps.
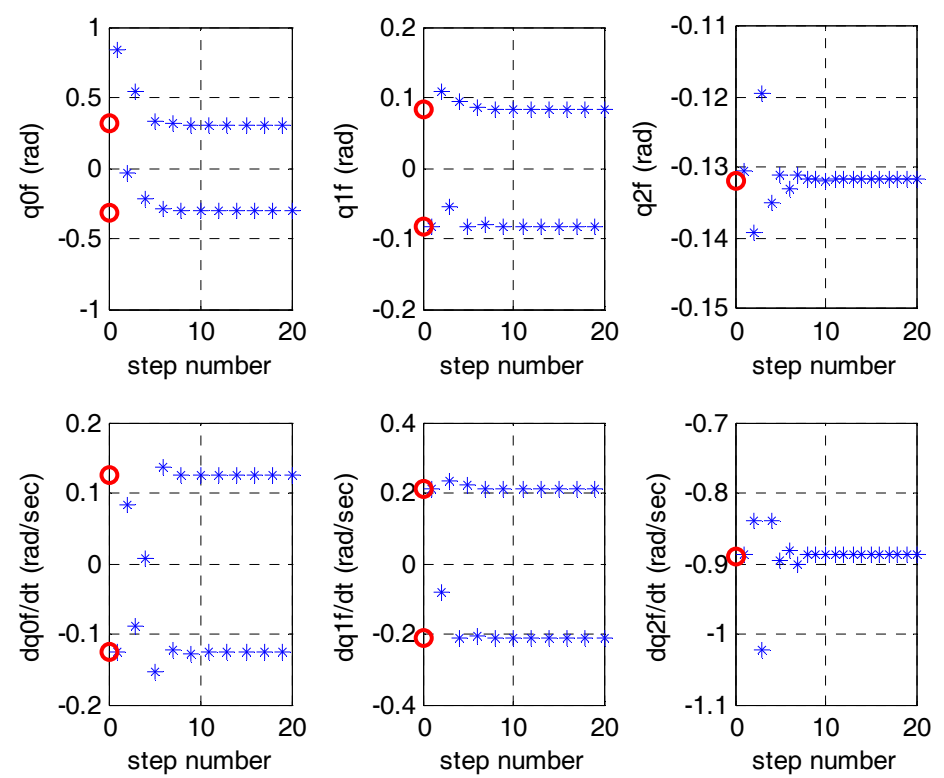

Fig. 9. Evolution of unactuated joints $\left(q_{0}, q_{1}, q_{2}\right)$ at the end of each step when a perturbation of $\pi / 6$ is added to $q_{0}$. The small circles represent the values on the desired periodic orbit. 

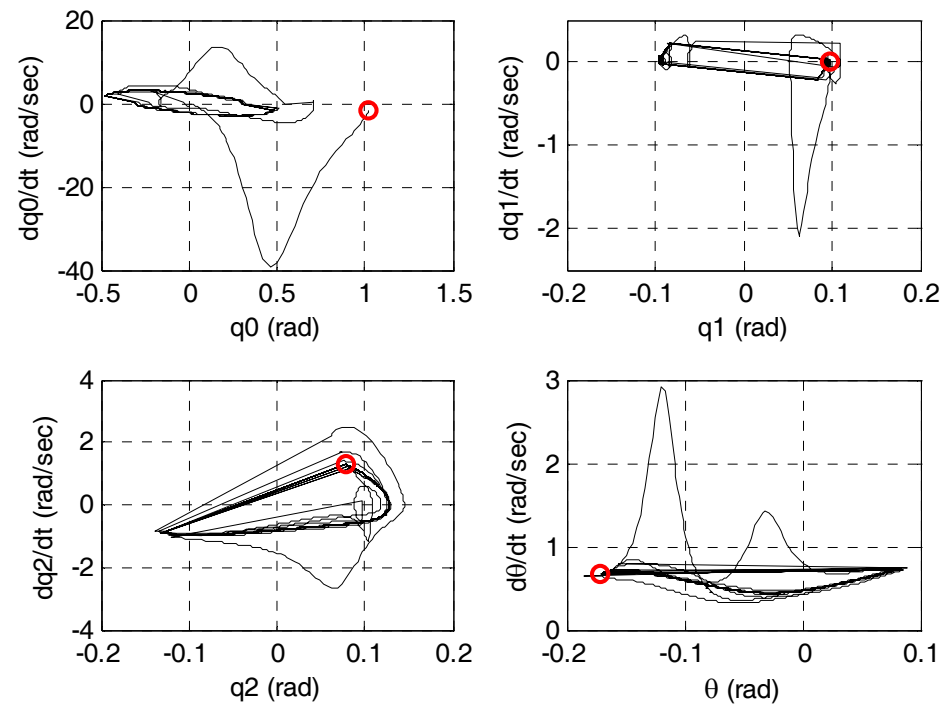

Fig. 10. Phase-plane plots for $\left(q_{0}, q_{1}, q_{2}, \theta\right)$ when a perturbation of $\pi / 6$ is added on the initial value of $q_{0}$. Each variable converges to the desired periodic motion. The small circles represent the initial state.

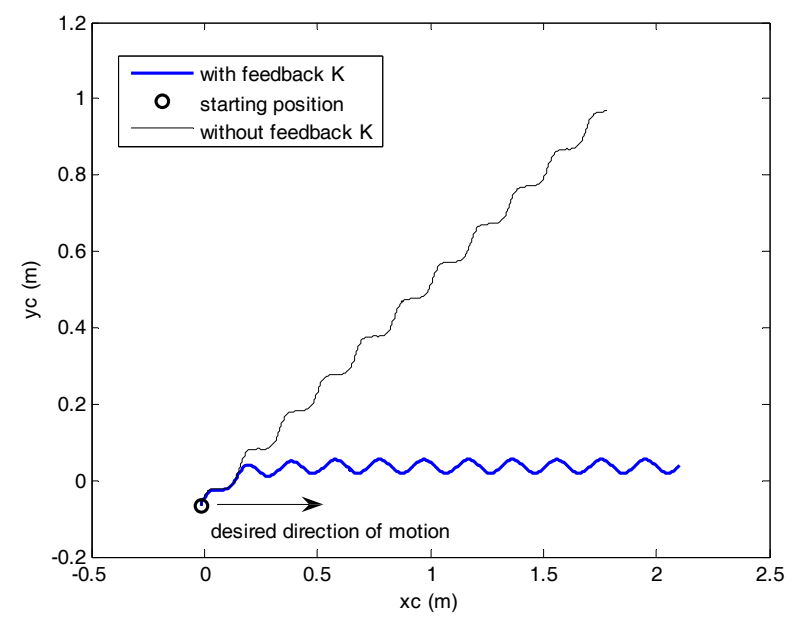

Fig. 11. Evolution of the center of mass in the $x-y$ plane for a perturbation of $\pi / 6$ is added on $q_{a}=\left[q_{3}, q_{4}, \cdots, q_{8}\right]^{T}$, cases of with and without stride-to-stride feedback control are shown. 


\section{Remark 3:}

The event-based controller developed in (22)-(24) holds the feedback correction $\beta$ constant over two steps. This is because the parameter $\beta$ is updated at each impact of leg-1 with the ground, consistent with the use of the Poincare map $P^{z}$. It is straightforward to provide feedback corrections with each leg impact. For simplicity, this is explained using the Poincare map of the full-order model (4), but applies equally well to the restricted Poincaré maps. The map $P: S_{2} \rightarrow S_{2}$ factors as noted before as $P=P_{12} \circ P_{21}$, with $P_{12}: S_{1} \rightarrow S_{2}$ and $P_{21}: S_{2} \rightarrow S_{1}$, where here we have ignored any dependence on $\beta$. The maps $P_{12}$ and $P_{21}$ define a periodically time-varying control system with period-2 via

$$
x_{k+1}=P_{i(k)}\left(x_{k}, \beta_{k}\right)
$$

where, $i(k)=12$ for $k$ odd and $i(k)=21$ for $k$ even. Analogous to (22) and (23), the Jacobian linearization of the system (25) can be computed on the basis of a fixed point $x_{2}^{*}=P\left(x_{2}^{*}, 0\right) \in S_{2}$ and $x_{1}^{*}=P_{12}\left(x_{2}^{*}, 0\right) \in S_{1}$ yielding a linear-time varying, period-2 control system

$$
\delta x_{k+1}=A(k) \delta x_{k}+F(k) \beta_{k} .
$$

The solution of an LQR problem with constant weights yields a time-varying, period-2 feedback $K_{k}$, replacing the constant gain matrix used in (24). 


\section{Steering Along a Curved Path}

This section modifies the stride-to-stride controller in order to achieve steering along a desired direction. The controller is then enhanced to achieve steering along a desired path of low curvature. The results are based on two properties of the closed-loop system designed in Sections 3 and 4. The first property is the inherent total stability, or what is now called in the control literature (local) input-to-state stability $^{16}$, of exponentially stable fixed points. The second property is a feedback symmetry ${ }^{14}$ that exists with respect to changes in the desired yaw.

\subsection{Stability Properties}

We return to the interpretation of a Poincaré map as a time-invariant discrete-time control system evolving on the Poincare section $S_{2}$. Extension to a period-2 time-varying control system is possible as in Remark 3.

Consider $P: S_{2} \times \mathrm{B}_{0} \rightarrow S_{2}$ with the associated control system introduced in (22)

$$
x_{k+1}=P\left(x_{k}, \beta_{k}\right)
$$

and feedback in (23). Define

$$
\bar{P}\left(x, x^{*}\right)=P\left(x,-K\left(x-x^{*}\right)\right),
$$

and let $\Phi: G \times Q \rightarrow Q$ be the group action on the configuration space $Q$ (based on yaw rotation) introduced in Section 2.4, and let $\Psi$ be its lift to the state space $T Q$. 
Proposition 5: For all $g \in G$,

$$
\Psi_{g} \circ \bar{P}\left(x, x^{*}\right)=\bar{P}\left(\Psi_{g}(x), \Psi_{g}\left(x^{*}\right)\right)
$$

and consequently,

1) $\Psi_{g}\left(x^{*}\right)$ is a fixed point of

$$
x_{k+1}=\bar{P}\left(x, \Psi_{g}\left(x^{*}\right)\right)
$$

2) the linearization of (30) about the fixed point $\Psi_{g}\left(x^{*}\right)$ is independent of $g$, and

thus if $K$ in (28) exponentially stabilizes the nominal fixed point $x^{*}$, it also stabilizes $\Psi_{g}\left(x^{*}\right)$.

Proof:

We start by noting that for all $g \in G, \Psi_{g}(x)-\Psi_{g}\left(x^{*}\right)=x-x^{*}$, and for all $\beta$, $\Psi_{g} \circ P(x, \beta)=P\left(\Psi_{g}(x), \beta\right)$, where the later holds in particular for $\beta=-K\left(x-x^{*}\right)$.

Hence,

$$
\begin{aligned}
\bar{P}\left(\Psi_{g}(x), \Psi_{g}\left(x^{*}\right)\right) & =P\left(\Psi_{g}(x),-K\left(\Psi_{g}(x)-\Psi_{g}\left(x^{*}\right)\right)\right) \\
& =P\left(\Psi_{g}(x),-K\left(x-x^{*}\right)\right) \\
& =\Psi_{g} \circ P\left(x,-K\left(x-x^{*}\right)\right) \\
& =\Psi_{g} \circ \bar{P}\left(x, x^{*}\right)
\end{aligned}
$$

proving (29). Part (1) is immediate and part (2) holds because, for all $g$, the Jacobian of $\Psi_{g}(x)$ with respect to $x$ is the identity; see (10).

It is important to note that if the nominal fixed point $x^{*}$ corresponds to walking 
parallel to the x-axis, for example, then $\Psi_{g}\left(x^{*}\right)$ corresponds to walking at an angle $g$ with respect to the x-axis. We now wish to treat the desired yaw angle $g$ as an input to the control system, and vary it "step-to-step" in order to steer the robot. With this in mind, consider the system

$$
x_{k+1}=\widetilde{P}\left(x, g_{k}\right)=\bar{P}\left(x, \Psi_{g}\left(x^{*}\right)\right)
$$

The new function $\widetilde{P}(x, g)$ is introduced to make explicit the use of the yaw-direction $g \in G$ as a variable that can be changed event-to-event. The control action rotates the set point in (27) and (28), which results in the rotation of the robot, that is, steering. The next result describes the stability properties of the steering process.

Proposition 6 (Local input-to-state stability) :

For every $\varepsilon_{1}>0$, there exist $\delta_{1}>0$ and $\delta_{2}>0$ such that, for every $g \in G$, every initial condition satisfying $\left\|x_{0}-\Psi_{g}\left(x^{*}\right)\right\| \leq \delta_{1}$ and all input sequences satisfying $\left\|g_{k}-g\right\| \leq \delta_{2}$, the solution of (31) exists for all $k>0$ and satisfies

$$
\left\|x_{k}-\Psi_{g}\left(x^{*}\right)\right\| \leq \varepsilon_{1}
$$

If, in addition to the above, the input sequence $g_{k}$ converges to $\bar{g}$, then the state converges to $\Psi_{\bar{g}}\left(x^{*}\right)$; that is,

$$
g_{k} \rightarrow \bar{g} \Rightarrow \lim _{k \rightarrow \infty}\left\|x_{k}-\Psi_{\bar{g}}\left(x^{*}\right)\right\|=0
$$

Proof: 
These properties are immediate from restricting the input-to-state stability (ISS) theorems of Jiang and Wang ${ }^{16}$ to an open neighborhood of the equilibrium. In particular, Example 3.4 of Jiang and Wang ${ }^{16}$ shows that exponential stability of the linearization implies the existence of a quadratic ISS-Lyapunov function about an open neighborhood of the equilibrium point, and then Lemma 3.5 of Jiang and Wang ${ }^{16}$ establishes input-to-state stability.

Q.E.D

In words, the first part of Proposition 6 states that small changes in desired rotation will not destabilize the robot. The second part states that if the commanded rotation settles to a constant value, the robot will asymptotically settle to a new heading corresponding to the commanded rotation, say $\bar{g}$. At this point, the first part of Proposition 6 applies again, so the robot can be further rotated; moreover, by Proposition 5, the linearization about the new equilibrium point $\Psi_{\bar{g}}\left(x^{*}\right)$ does not depend on $\bar{g}$, so the rate of convergence to the equilibrium is uniform in $\bar{g}$. From this, it follows that there exits $\delta_{3}>0$ such, if $\left|g_{k+1}-g_{k}\right| \leq \delta_{3}$, the robot will turn and not fall. This will be demonstrated in simulations in the next section.

\subsection{Stride-to-Stride Controller for Controlling Orientation}

Proposition 6 can be used to plan a turning motion for the bipedal robot. The change 
of orientation can be implemented through a change of the desired fixed point at each step, and as a consequence, through a modification of the reference trajectory for the controlled output via (24). The stride-to-stride controller is modified as

$$
\beta_{k}=-K\left(x_{k}^{z}-\Psi_{g_{k}}\left(x^{z^{*}}\right)\right)=-K\left(x_{k}^{z}-x^{z^{*}}-g_{k} e_{1}\right)=-K\left(\delta x_{k}^{z}-g_{k} e_{1}\right),
$$

with $e_{1}=\left[\begin{array}{llll}1 & 0 & \cdots & 0\end{array}\right]^{T}$ and $g_{k}$ is the desired absolute yaw rotation. If $g_{k}$ is slowly varied step to step, then the robot can execute a more complex path. The feedback gain $K$ distributes changes to all of the actuated joints as needed for achieving turning.

In order that the robot's motion will converge to a circular path, the desired angle $g_{k}$ can be modified by a constant value at each step per $g_{k+1}=g_{k}+\alpha$. As an illustration, to induce the $3 \mathrm{D}$ point feet bipedal robot to follow a circle in the counter-clockwise direction, the commanded value of $g_{k}$ was incremented by $\alpha=0.1 \mathrm{rad}$. at each leg touchdown. Fig. 12 shows the evolution of the final values of the uncontrolled variables $\left(q_{0}, q_{1}, q_{2}\right)$ from one step to the next. These variables update automatically to new command values in order to follow a circular path. Fig. 13 shows phase-plane plots of $\left(q_{0}, q_{1}, q_{2}, \theta\right)$. Fig. 14 shows the evolution of the center of mass in the $\mathrm{x}-\mathrm{y}$ plane; the radius of the circle is about $1.0 \mathrm{~m}$ and it takes 30 seconds to complete one lap of the circle. 

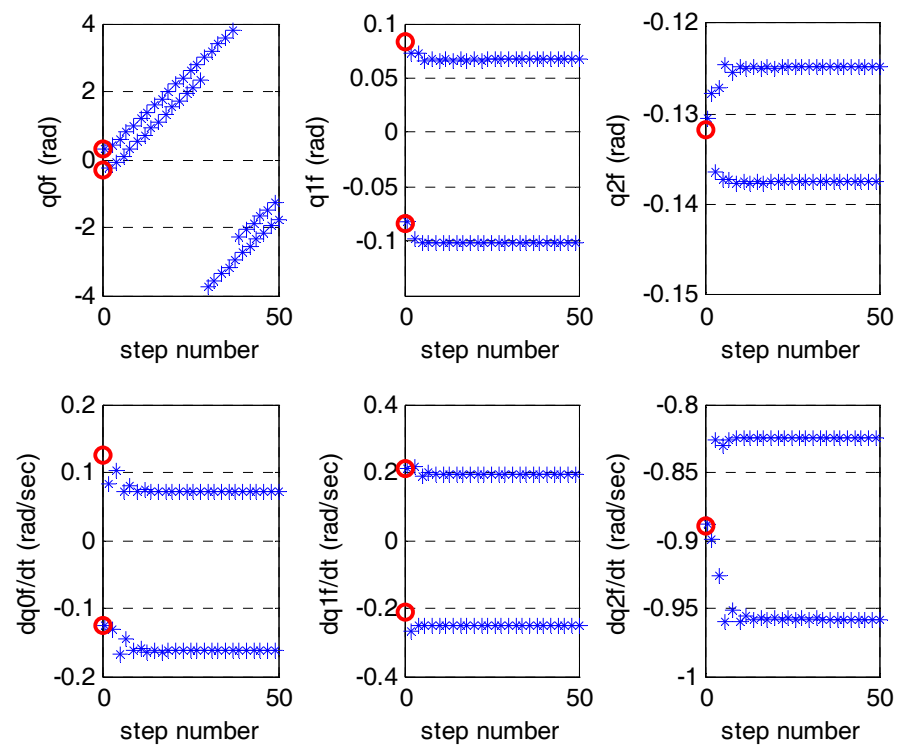

Fig. 12. Evolution of unactuated joints $\left(q_{0}, q_{1}, q_{2}\right)$ at the end of each step when the robot changes commanded direction at each step in order to follow a circle. The small circles represent the values on the desired periodic orbit.
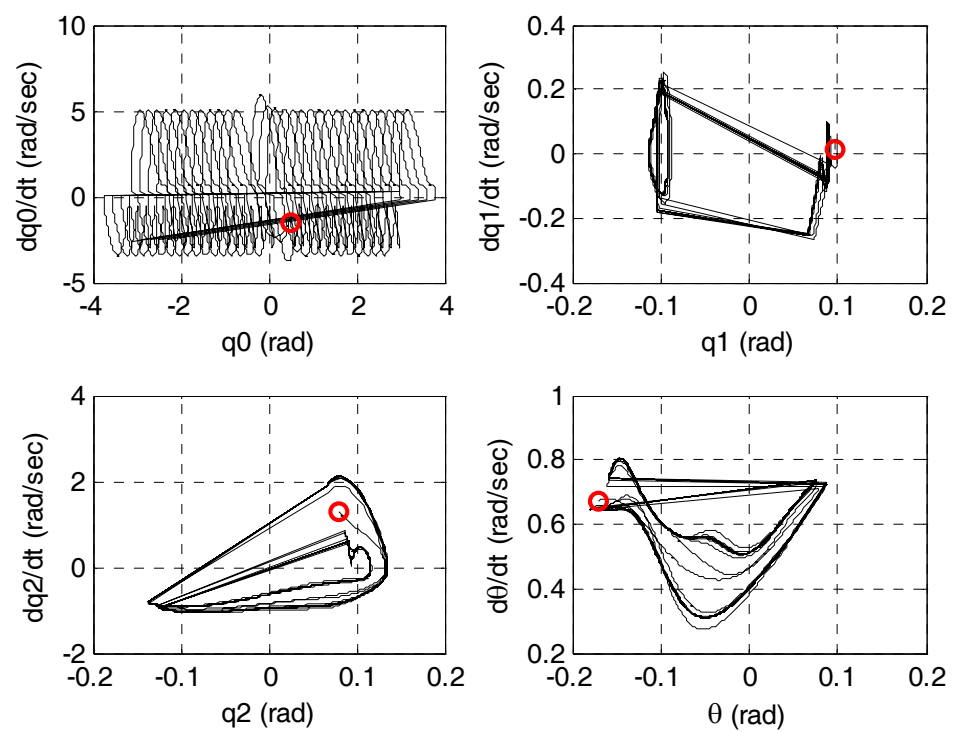

Fig. 13. Phase-plane plots of $\left(q_{0}, q_{1}, q_{2}, \theta\right)$ when the robot changes commanded direction at each step in order to follow a circle; variable $q_{0}$ steps through $360^{\circ}$. The small circles represent the initial state. 


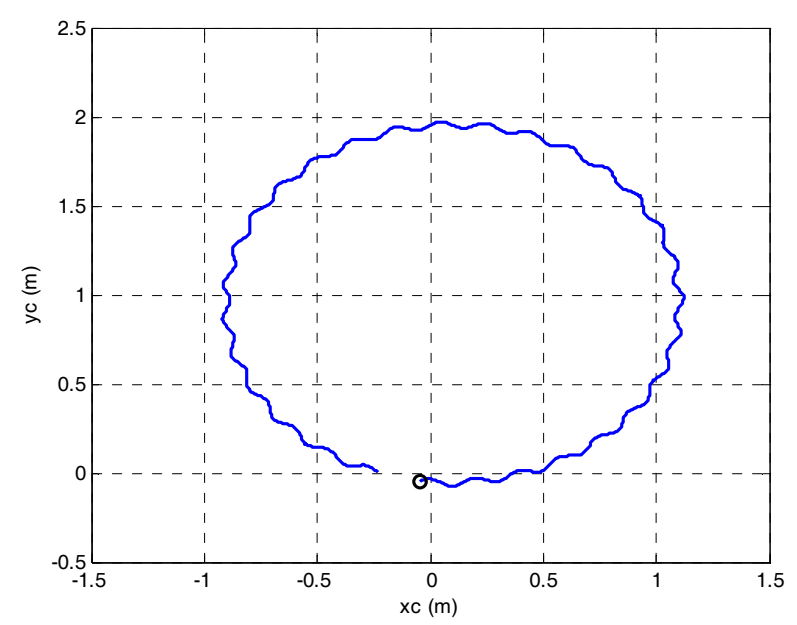

Fig. 14. Evolution of the center of mass in the $x-y$ plane for that the robot changes direction of following a circular path, where the small circle denotes the starting position.

\subsection{Stride-to-Stride Controller for Motion Along a Desired Path in the World Frame}

In Fig. 11, the stride-to-stride controller (24) can only stabilize the orientation angle of the walking direction but leaves the y-component of the COM (center of mass) uncontrolled. A high-level supervisory control can be integrated into the overall controller to resolve this problem. For example, suppose that it is desired to steer the robot's COM along a path consisting of the world-frame's x-axis, $\theta_{r}=0$, with $y=y_{r}$. Let $\left[x_{c}, y_{c}, z_{c}\right]^{T}$ be the mass center of the robot, a simple strategy to realize this goal is to augment the stride-to-stride control law (24) with an additional proportional correction term $\gamma_{k}$,

$$
\beta_{k}=-K\left(\delta x_{k}^{z}-\gamma_{k} e_{1}\right),
$$


where

$$
\gamma_{k}=\left\{\begin{array}{cc}
Q_{0} & k_{1}\left(y_{r}-y_{c}\right)>Q_{0} \\
-Q_{0} & k_{1}\left(y_{r}-y_{c}\right)<-Q_{0}, \\
k_{1}\left(y_{r}-y_{c}\right) & \text { otherwise }
\end{array}\right.
$$

with a proportional gain $k_{1}$ and a saturation level $Q_{0}$ in order to take into account that the amount of turning that can be realized in one step is limited. Fig. 15 shows the evolution of the COM in the x-y plane for this example (Case 1). The robot not only converges to the orientation angle of the $\mathrm{x}$-axis but also controls its $\mathrm{y}$-coordinate of its COM to within a small range of $y=y_{r}=0$. Fig. 16 provides an expanded view of the evolution of the COM.

In the next example, it is desired that the robot move along a path consisting of the world-frame's y-axis, $\theta_{r}=\pi / 2$, at the location of $x=x_{r}$. With a similar supervisory steering control strategy, the stride-to-stride controller (34) for controlling robot's orientation is also augmented with an additional term as shown below

$$
\beta_{k}=-K\left(\delta x_{k}^{z}-\left(\theta_{k}+\gamma_{k}\right) e_{1}\right)
$$

in which $\theta_{k}$ is the desired orientation angle of the motion at step $k$,

$$
\theta_{k}=\sum_{i=1}^{k} \alpha_{i}
$$

where

$$
\alpha_{k}=\left\{\begin{array}{cc}
Q_{0} \quad k_{0}\left(\theta_{r}-\theta_{k-1}\right)>Q_{0} \\
-Q_{0} \quad k_{0}\left(\theta_{r}-\theta_{k-1}\right)<-Q_{0} \\
k_{0}\left(\theta_{r}-\theta_{k-1}\right) \quad \text { otherwise }
\end{array}\right.
$$


and $k_{0}$ is a constant gain. The position correction term $\gamma_{k}$ is a proportional control with saturation

$$
\gamma_{k}=\left\{\begin{array}{cc}
Q_{0} & k_{1}\left(x_{c}-x_{r}\right)>Q_{0} \\
-Q_{0} & k_{1}\left(x_{c}-x_{r}\right)<-Q_{0} \\
k_{1}\left(x_{c}-x_{r}\right) & \text { otherwise }
\end{array}\right.
$$

Fig. 16 also shows the evolution of the COM in the $\mathrm{x}-\mathrm{y}$ plane for the above example (Case 2). The robot not only turns to the orientation angle of the y-axis but also controls the X-coordinate of its COM to within a small range of $x=x_{r}=1.0$.

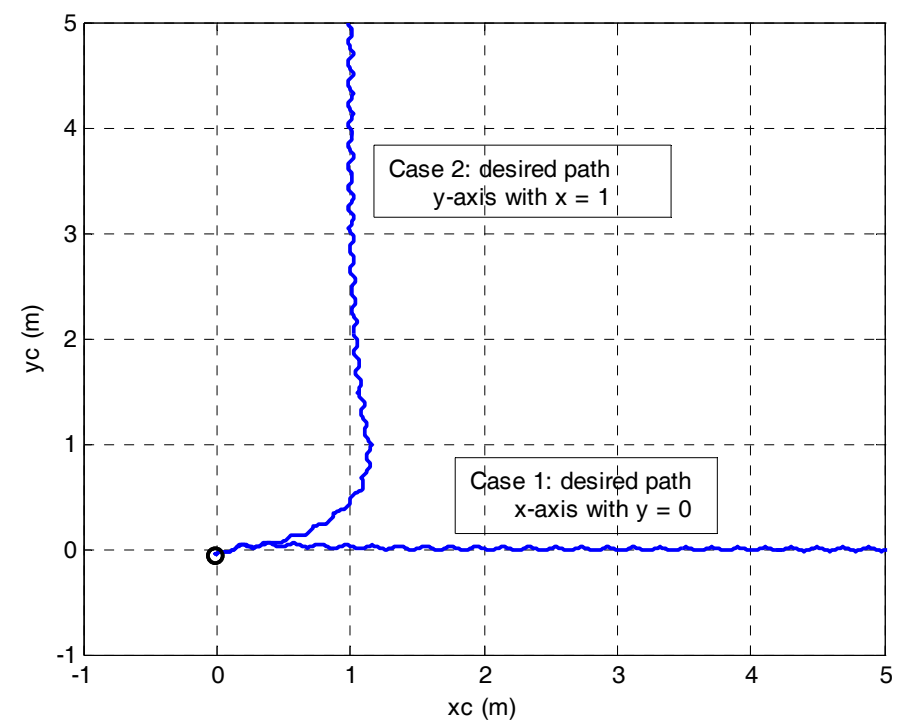

Fig. 15. Evolution of the center of mass in the $x-y$ plane under steering control. Case 1: along a path of the x-axis in the world frame, and Case 2: along a path of the world frame's $y$-axis at location of $x=1$. The small circle denotes the starting position and is the same as in Fig. 11. 


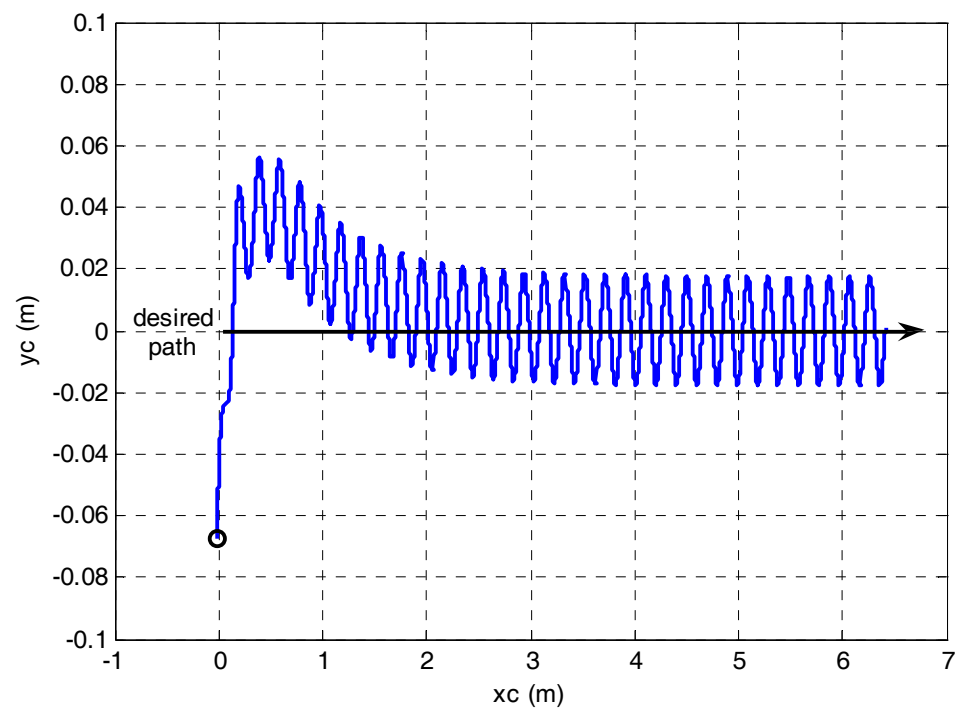

Fig. 16. Enlarged version of Case 1 in Fig. 15.

\section{Conclusion}

A 3D point-feet bipedal model has been studied, with the objective of steering the robot in addition to creating a stable walking motion. The model assumed rigid links, a passive 3 DoF point contact between the stance leg end and the ground, with all other degrees of freedom actuated. The controller design exploits a natural symmetry present in a $3 \mathrm{D}$ robot $^{14}$ in order to achieve asymptotically stable steering. The method of virtual constraints was first used to design a time-invariant, within-stride feedback controller that stabilized all but the yaw motion of the robot. The closed-loop system (i.e., robot plus controller) was shown to be equivariant under yaw rotations. A supplemental event-based feedback controller was then designed that asymptotically stabilized the yaw motion, resulting in the existence of exponentially stable periodic 
orbits in the closed-loop hybrid system.

The symmetry property was used to establish that the supplemental controller provided local, input-to-state stability that is uniform in the desired yaw (steering) angle. By adjusting the set point of the event-based controller, it was possible even to direct the motion of its center of mass along a given path. This was achieved without designing a new periodic orbit for turning. Instead, the controller could be designed on the basis of a single motion designed for walking in a straight line. The event-based controller distributes commands to all of the actuated joints in order to achieve a sufficiently small, desired amount of turning. The restriction on the amount of rotation that can be achieved in a single step arises from the fact that the nominal periodic orbit of the closed-loop system is only locally exponentially stable.

A more energy efficient modification of the actuated joints could probably be proposed if a change in the impact configuration is allowed; this was not studied here. A controller similar to the one developed in this paper is applicable to the model treated in Chevallereau et al. ${ }^{1}$, which assumed a passive 2 DoF point contact between the stance leg end and the ground, with no yaw motion. In this case, the change of the yaw angle comes only from the impact phase, when the stance leg changes, and not from the single support phase; nevertheless, a similar strategy of steering control and stability analysis can be developed. 
There are several ways in which the result can be extended. To achieve turning with a more aggressive turning rate, solutions of the model can be specifically designed to achieve a large amount of turning in one step. These solutions could then be pieced together as in Westervelt et al. ${ }^{15}$ to achieve maneuvers that steer the robot around obstacles. Treating a model without feet may make it difficult to design controllers that allow the robot to stop, take a step backward and redirect its motion. Hence, another interesting extension of the control strategy developed here is to consider a model with feet and to compare with ZMP based methods ${ }^{18,19}$.

\section{Acknowledgments}

This work of C.L. Shih was supported by the Taiwan National Science Council (NSC) under Grant NSC 97-2212-E-011-062. The work of J.W. Grizzle is supported by NSF grant ECS-0600869.

\section{References}

1. C. Chevallereau, J.W. Grizzle and C.L. Shih, "Asymptotically stable walking of a five-Link underactuated 3D bipedal robot," IEEE Transactions on Robotics 25, pp. $37-50$ (2009). 
2. Y. Sakagami, R. Watanabe, C. Aoyama, S. Matsunaga, N. Higaki, and K. Fujimura, "The intelligent ASIMO system overview and integration," Proceeding of the 2002 IEEE/RSJ International Conference on Intelligent Robots and Systems, EPFL Lausanne, Suisse (2002) pp. 2478-2483.

3. M. Yagi and V. Lumelsky, "Synthesis of turning pattern trajectories for a biped robot in a scene with obstacles," Proceedings of the 2000 IEEE/RSJ International Conference on Intelligent Robots and Systems, (2000) pp. 1161-1166.

4. K. Miura, S. Nakaoka, M. Morisawa, K. Harada, and S. Kajita, “A friction based twirl for biped robots," IEEE-RAS International Conference on Humanoid Robots, (Dec. 2008) pp. 279-284.

5. S. Aoi, K. Tsuchiya, and K. Tsujita, "Turning control of a biped locomotion robot using nonlinear oscillators," Proceeding of the 2004 IEEE International Conference on Robotics and Automation, New Orleans, LA (April 2004) pp. 3043-3048.

6. K. Nishiwak, S. Katoshi, J. Kuffner, M. Inaba, and H. Inoue, “Online humanoid walking control system and a moving goal tracking experiment," Proceedings of the 2003 IEEE International Conference on Robotics and Automation, Taipei, Taiwan, (Sep. 2003) pp. 911-916. 
7. R. Kurazume, T. Hasegawa and K. Yoneda, "The sway compensation trajectory for a biped robot," Proceedings of the 2003 IEEE International Conference on Robotics and Automation, Taipei, Taiwan (Sep. 2003) pp. 925-931.

8. R.D. Gregg and M.W. Spong, "Reduction-based control with application to three-dimensional bipedal walking robots," Proceedings of the 2008 American Control Conference, Seattle, WA (2008).

9. R.D. Gregg and M.W. Spong, "Reduction-based control of three-dimensional bipedal walking robots," International Journal of Robotics Research, to appear. Pre-print available at http://ijr.sagepub.com/cgi/content/abstract/0278364909104296v1

10. R.D. Gregg and M.W. Spong, "Reduction-based control of branched chains: application to three-dimensional bipedal torso robots," IEEE Conference on Decision and Control, Shanghai, China (2009).

11. R.D. Gregg and M.W. Spong, "Bringing the compass-gait bipedal walker to three dimensions," International Conference on Intelligent Robots and Systems, St. Louis, MO (2009).

12. C.L. Shih, J.W. Grizzle, and C. Chevallereau, "Asymptotically stable walking of a simple underactuated 3D bipedal robot," The $33^{\text {rd }}$ Annual Conference of the IEEE 
Industrial Electronics Society (IECON), Taipei, Taiwan (Nov. 2007) pp.2766-2771.

13. E.R. Westervelt, J.W. Grizzle, C. Chevallereau, J. Choi, and B. Morris, Feedback control of dynamic bipedal locomotion, CRC Press, Boca Raton FL (June 2007).

14. M.W. Spong and F. Bullo, "Controlled symmetries and passive walking," IEEE Transactions on Automatic Control 50, pp. 1025-1031 (July, 2005).

15. E.R. Westervelt, J.W. Grizzle, and C. Canudas de Wit, "Switching and PI Control for Planar Biped Walkers," IEEE Transactions on Automatic Control 48, pp. 308-312 (Feb. 2003).

16. Z.P. Jiang and Y. Wang, "Input-to-State stability for discrete-time nonlinear systems," Automatica, 37, pp. 857-869 (2001).

17. B. Morris and J.W. Grizzle, "Hybrid invariant manifolds in systems with Impulse effects with application to periodic locomotion in biped robots," IEEE Transactions on Automatic Control 54, pp. 1751-1764 (Aug. 2009).

18. R.P. Kumar, J. Yoon, and G. Kim, "The simple passive dynamics walking model with toed feet: a parametric study," Robotica 27, pp. 701-713 (2009).

19. T.-Y. Wu and T.-J. Yeh, "Optimal design and implementation of an energy-efficient biped walking in semi-active manner," Robotica 27, pp. 841-852 (2009). 